\title{
LA ENSEÑANZA MEDIA DE LA FILOSOFÍA EN LAS ACTUALES CONDICIONES CULTURALES Y SOCIALES DE NUESTROS PAÍSES
}

\author{
Ricardo Navia(1)
}

Resumen: Esta ponencia comienza por señalar que los cursos de Filosofía en la enseñanza media constituyen una instancia insustituible en la estructuración de una actitud mínimamente crítica que es imprescindible para la participación responsable y libre en una sociedad democrática. Desde el punto de vista metodológico, se considera que los grandes temas de la enseñanza media de la Filosofía no se resuelven meramente con tecnología pedagógica sino fundamentalmente con análisis y reflexión sobre las condiciones sociales, culturales e ideológicas que rodean $e$ inciden sobre la educación y principalmente, sobre la enseñanza de esta actividad crítica, radical y globalizadota, que es la Filosofía. Mostramos que en el momento actual existe en nuestra América una situación con varias características que están determinando una dificultad creciente para el logro de los importantes objetivos de la enseñanza media de la filosofía. Lo que nos proponemos es analizar esas condiciones y mostrar luego que también existen "contra-condiciones" que podrían posibilitarnos un relanzamiento de nuestra actividad.

Las condiciones negativas provienen fundamentalmente del empobrecimiento material y simbólico a que ha sido sometido el sistema educativo, de la trivial politización y burocratización del aparato educativo, de las condiciones socioculturales de los educandos, del empobrecido entorno cultural de los medios de difusión masiva y del adelgazamiento de la discusión política e ideológica. Características de un paradójico posmodernismo en las tierras del subdesarrollo. Todos estos rasgos dificultan enormemente el proceso educativo, y la enseñanza de la filosofía queda especialmente comprometida. En esa enseñanza secundaria, a la larga, la enseñanza de la filosofía estaría condenada a desaparecer.

La pregunta clave es hoy, ¿existe frente a la pedagogía light, neo-conservadora y estratificada otra alternativa que rejerarquice la educación en general y la enseñanza de la filosofía en particular? ¿Puede existir una tal posibilidad en medio de las condiciones descriptas?

Mi parecer es que no hay una respuesta segura para esta pregunta crucial. La educación en general y la enseñanza de la filosofía como "barómetro" privilegiado de ésta, son enormemente sensibles a múltiples factores sociales, económicos, políticos, culturales e ideológicos, cuyo desarrollo no puede ser previsto. Sin 
embargo, relevamos también algunos signos en lo social y en lo político que podrían estar posibilitando un giro dialéctico hacia un reposicionamiento progresista de la educación en general y de la enseñanza de la filosofía en particular.

A la hora de visualizar las direcciones en que podemos contribuir a un desenlace positivo de esta situación distinguimos entre propuestas que hacen a la educación media como un todo y propuestas que hacen a la enseñanza filosófica en particular. Dentro de los últimas, tres de las propuestas se dirigen a conseguir una revalorización de los cursos de filosofía por parte de los alumnos, y la cuarta apunta además a rejerarquizar a la Filosofía en el contexto del currículo y en el seno mismo de la comunidad educativa. Es que: ninguna campaña "reivindicadora" logrará convencer a la opinión pública ni a las autoridades de la importancia de la enseñanza media de la filosofía, si antes no se la hicimos sentir a los propios alumnos y a los propios colegas de otras asignaturas.

Hace ya unos 25 años que me inicié como de profesor de Filosofía a nivel medio. A pesar de que en los últimos años no he tenido grupos directamente a mi cargo, la actuación como formador y supervisor de las nuevas generaciones de docentes me permite hacer una evaluación de la actividad con ese margen temporal, donde varias condiciones, que han ido cambiando, merecen nuestro análisis.

Si bien considero que lo que a uno más lo determina en la elección de una profesión no es su valor social sino la curiosidad y el placer íntimo que le proporciona moverse dentro de ciertos temas, veía entonces, -y veo ahora- la enorme importancia formativa de cursos medios de Filosofía para todos los jóvenes, vayan o no a cursar carreras universitarias, sean estas humanísticas, científicas o artísticas. Sucede que los cursos secundarios de Filosofía satisfactoriamente logrados constituyen una instancia que no tiene sucedáneos en el currículo. En efecto, no hay ninguna otra instancia donde se reflexione sobre el fundamento y los límites del conocimiento, tratando de generar criterios sobre la distinción entre conocimiento fundamentado y no fundamentado y de despejar tanto el oscurantismo como la mistificación de la ciencia; ningún otro ámbito donde se reflexione sobre problemas éticos, estéticos, antropológicos, sociohistóricos y culturales, procurando un antídoto contra el dogmatismo, el fanatismo y la intolerancia. Una instancia, además, donde se desarrollan las capacidades de argumentación y discusión de ideas explícitamente fundamentadas y con elucidación de los principios y supuestos implicados, como modelo privilegiado de cualquier análisis, elucidación y evaluación de supuestos de un discurso que involucre principios generales.

Todo ello hace de los cursos de Filosofía una instancia insustituible en la estructuración de una actitud de apertura mental, de análisis, de fundamentación y de exigencia de fundamentación, que son imprescindibles para la participación responsable y libre en una sociedad democrática.

Por cierto que esa actitud no la genera sólo la Filosofía sino la educación en su conjunto, pero la enseñanza de la Filosofía actúa como una instancia 
cohesionadora, explicitadora y reforzadora de esas capacidades. Me animaría a decir que: sin Filosofía, algo importante faltaría en la formación de un ciudadano libre en una democracia moderna, que no lo quiera ser sólo de modo formal. Por todo ello, creo que reflexionar sobre las modalidades, los resultados y las condiciones de esa práctica constituye un tema de máxima importancia cultural, social y política. Y es también él, un tema filosófico.

Fue esa preocupación la que hace casi quince años me motivaba para escribir un entusiasta artículo - que se publicó en la revista de educación de la PUC de San Pablo con el cual comencé mi relación académica con Brasil - sobre "La enseñanza media de la filosofía y los grandes temas de nuestro tiempo", y hoy me vuelve a convocar con parecido objetivo. Pero, por supuesto: "nuevas aguas corren tras las aguas" y "nunca entramos dos veces en el mismo río". Las condiciones sociales y culturales de América y sobre todo del mundo han cambiado en diversos sentidos.

Por experiencia y convicción, considero que los grandes temas de la enseñanza media de la Filosofía no se resuelven con tecnología pedagógica sino con reflexión y formación filosóficas. Y muy especialmente con análisis y reflexión sobre las condiciones sociales, culturales e ideológicas que rodean $e$ inciden sobre la educación y muy principalmente sobre la enseñanza de esta actividad crítica radical y globalizadora que es la Filosofía.

En 1988 los países del cono sur salíamos de la larga noche del autoritarismo y el aislamiento cultural internacional y de un período muy oscuro de la educación que debíamos reconectar con un mundo que había seguido cambiando y produciendo, a la par de evitar ciertas tendencias de trivialización de la enseñanza que se avizoraban en algunos cuadros oficiales de las democracias reconquistadas. En ese contexto, nuestra propuesta en aquel momento giraba en torno a tres ideas básicas:

- Que la enseñanza media de la filosofía no podía dejar de abordar lo que llamábamos los grandes temas de nuestro tiempo.

- Que la enseñanza secundaria de la filosofía debía hacer especial hincapié en ubicar los problemas filosóficos en su contexto histórico-cultural de surgimiento y vigencia, como forma privilegiada de acercarse a reconstruir y transmitir su sentido y alcance.

- Que no se debía tratar de resolver los problemas de la enseñanza media de la filosofía, ni por una trivialización de los contenidos y los materiales, ni por una sacralización de supuestos métodos de participación y dinamización forzada de la actividad en el aula, que a veces terminaban derivando en una rebaja de los contenidos y en una infantilización de la actitud de los alumnos.

Quince años después, existe en el mundo y muy especialmente en nuestra América una situación con algunas características nuevas y otras que se han agravado. Características que prima facie estarían determinando una dificultad creciente para el logro de los importantes objetivos de la enseñanza media de la filosofía. Lo que 
aquí nos proponemos es analizar esas condiciones y mostrar luego que también existen "contra-condiciones" que podrían posibilitarnos un relanzamiento de nuestra actividad.

\section{Condiciones adversas}

En el momento actual del mundo y de América Latina existen varias y diversas condiciones que dificultan el logro de los objetivos de la enseñanza secundaria de la Filosofía.

En primer lugar, están las pésimas condiciones materiales en que se desarrolla la enseñanza media pública, un escenario que se conforma a través de: locales inadecuados, falta de materiales y de libros, grupos superpoblados, docentes pésimamente remunerados y multiempleados que carecen de tiempo para actualización y adecuado seguimiento de sus alumnos. Eso es lo primero, y no es poco. Quienes intentan desconocerlo argumentando por el lado de la "vocación" o el "apostolado" o son hipócritas o son ignorantes de las condiciones mínimas del profesionalismo. Todo ello sin contar el efecto simbólico de trabajar en un entorno deprivado donde el ámbito educativo y el propio docente pierden su imagen paradigmática y esto se proyecta sobre la propia actividad y sus objetivos que -en el "imaginario social" resultan desprestigiados.

Al empobrecimiento material del sistema educativo estatal hay que agregar la distorsión del mismo originado por otros factores institucionales sobreagregados.

En primer lugar se ha producido una burocratización de la estructura educativa, no en el sentido de exceso de funcionarios - que por cierto los educativamente necesarios (docente de apoyo, psicólogos, etc.) escasean- sino en el sentido de que los funcionarios políticos y administrativos suelen tener mayor poder de decisión que los docentes y que los supervisores docentes de carrera.

También hemos presenciado una politización de los cargos de dirección educativa, que no se confían a docentes destacados sino a cuadros político-partidarios (muchas veces frustrados de "aspiraciones mayores") y una distorsión de la carrera docente en el sentido de que los altos cargos de planificación y supervisión no se dirimen por concurso de méritos intelectuales independientes sino por adhesión a planes impuestos o por méritos administrativos (los "nuevos gestores de la educación").

Estas distorsiones, que afectan todo el proceso educativo, inciden especialmente sobre la enseñanza de la Filosofía. No es difícil imaginar los múltiples errores en que puede incurrir un supervisor docente, de una tarea tan compleja como es la enseñanza de la Filosofía, cuando no es desempeñada por alguien que ha accedido a esa posición por su formación, su experiencia y sus aportes independientes, si no por simples méritos administrativos o, por lo receptivo que estos funcionarios pueden ser a los errores y simplismos de autoridades que, a su vez, no son docentes de sólida carrera. 
Por el lado de las condiciones socio-culturales de los educandos la situación es también adversa. Familias que no pueden costearse los materiales indispensables, algunos que no portan libros y cuadernos sino fotocopias aisladas en cuadernos polifuncionales; hogares sin bibliotecas mínimas ni diarios que introduzcan nociones de cultura general y habilidades lingüísticas mínimas, padres con jornadas extenuantes que no logran controlar, apoyar y motivar las tareas de sus hijos, etc. Padres exhaustos en medio de la amenaza constante de la desocupación que, lejos de poder apoyar la educación de sus hijos, más bien transmiten una depresión y un miedo al futuro que resulta inhibitoria de toda reflexión y toda tarea de largo aliento. Para confirmar la incidencia de estos factores alcanza con consultar los resultados de los sucesivos diagnósticos PISA (Programa para la Evaluación Internacional de los Estudiantes) sobre habilidades matemáticas, de lectura y de ciencias de los jóvenes de 15 años, auspiciados bianualmente por la UNESCO y la OCDE en 40 países del mundo, en los cuales siempre obtienen los primeros lugares países donde la educación tiene respaldo económico y autonomía técnica. O decir, que en Uruguay, un país que alguna vez se enorgulleció de su educación, hoy en día: de cada cien estudiantes que ingresan a la enseñanza media, solo 35 llegan a cumplir el ciclo completo de seis años.

Claro que también existen institutos secundarios privados: en un sector de los cuales se nuclean los hijos de la amenazada clase media y, en otros, los grupos sociales más beneficiados. Si bien en ambos casos consiguen condiciones educativas materialmente superiores, que, en general, aseguran resultados promediales algo superiores, no es claro que consigan eludir varios de los problemas antes reseñados; tanto porque no pueden evadirse de las condiciones culturales e ideológicas de la sociedad en su conjunto, como porque en la era de una economía globalizada y centralizada, también sus padres tienen lazos de dependencia con poderes mayores que le quitan interés por repensar los fundamentos y la globalidad.

Desde la esfera cultural el panorama -en principio- no es más alentador: carentes de libros, de las fundamentales enciclopedias (que conoció nuestra generación) y de diarios, los adolescentes -y sus padres- se sumergen en una TV de bajísimo nivel cultural, cultivadora de toda clase de simplismo e inmediatismo, donde incluso no escasean los mensajes contra-culturales y anti-intelectualistas. La apertura que podría significar el mundo informático está también desaprovechada: sin guías docentes que seleccionen y coordinen los "sites" de valor cultural, los jóvenes se sumergen en los sitios más banales de limitadísimos recursos culturales y lingüísticos o se limitan a una conversación empobrecida y anónima que antes desarrollaba de forma más enriquecedora. El efecto de este entorno "cultural" sobre la enseñanza de la Filosofía es también importante desde que ésta requiere una cierta base de cultura general y ciertas capacidades lingüísticas y de abstracción, que en estas condiciones tienden a ser sustituidas por un pensamiento concreto $e$ inmediatista, y un lenguaje empobrecido, con los cuales es muy difícil abordar problemas metateóricos o abstraer los fundamentos y los principios.

En el aspecto ideológico-político también se presentan algunas condiciones negativas: para empezar se ha producido un verdadero adelgazamiento de los 
espacios políticos; la discusión política en profundidad está casi totalmente ausente. Los grandes debates parlamentarios o ideológicos son muy esporádicos y tienden a ser sustituidos por acuerdos de cúpula, cuando no por resoluciones "obligadas" por las limitaciones sistémicas o las imposiciones desde los grandes centros de poder. Los ministros de economía son entrevistados por jóvenes profanos - jamás se contrata a los cientos de estudiantes de economía que buscan en vano un primer trabajo - para beneficio del señor ministro que contesta lo qué quiere, cómo quiere y cuándo quiere. Por cierto, a todo ello hay que agregar el efecto paralizante de la caída de las utopías que tiende a inhibir todo intento de crítica y de búsqueda de alternativas más o menos profundas.

También en este aspecto los efectos sobre la tarea filosófica son manifiestos: basta con decir que la filosofía es -de alguna manera- la profundización de un debate, del cual, históricamente, el debate político ha sido uno de sus ejemplos arquetípicos.

Estas son algunas de las condiciones que rodean al hecho educativo en nuestros estancados y dependientes países. Y aún habría que agregar las características emanadas de la peculiar traslación del posmodernismo y la globalización en las tierras del subdesarrollo, países que han ido adoptando algunos rasgos del neoliberalismo y han desandado gran parte de la legislación social y la economía regulada.

En realidad más que de una cultura del posmodernismo se trata de una cultura de la frustración reiterada de las promesas de cambio sobre una base de décadas de autoritarismo antipopular, que tiende a producir una especie de paradójico posmodernismo en sociedades desindustrializadas y fuertemente estratificadas.

Los cierto es que aparecen:

- una pérdida de los proyectos utópicos y colectivos que genera nihilismo, inmediatismo, individualismo, depresión y corrupción (el impúdico "hacé la tuya").

- una sensación real de que las grandes decisiones se toman en lejanos centros de poder que genera una paralizante sensación de impotencia y escepticismo

- pérdida del rol nivelador de la educación, desprestigio de la figura docente que ya no opera como modelo de identificación y depreciación del esfuerzo de largo plazo

- el hombre de los medios o el joven con olfato oportunista es el modelo de identificación difundido por los medios.

Por cierto aparecen también otras características compartidas con la posmodernidad: depresión, desmotivación, ansiedad, atención flotante (zapping), necesidad de satisfacción inmediata, etc.

Evidentemente, todos estos rasgos dificultan enormemente el proceso educativo al menos bajo los parámetros de la pedagogía de que disponemos, y, dado que la 
enseñanza de la filosofía resume incrementadas estas características, su enseñanza está especialmente comprometida.

\section{La respuesta light y conservadora}

Ante esta compleja situación, algunos docentes han intentado "huir hacia delante", hacia una enseñanza posmoderna: sustituyendo los textos clásicos por los comics, el lenguaje elaborado por la jerga juvenil, los problemas filosóficos por la última inquietud de los adolescentes, etc. Parecen ignorar que la actualización pedagógica es algo mucho más complejo, modesto y prudente. Habría que recordarles siempre que: "... correr tras los adolescentes no parece una solución para salvar la distancia con los mismos: son más veloces que los profesores" (1995, p. 125).

Otros, ante esta difícil situación, han aprovechado la necesidad de cambio para cambiar "hacia atrás". Acusando a los docentes de conservadores y apelando a las reivindicaciones de cambio de los jóvenes, han propuesto una educación desrregulada financiada no por el estado sino por los municipios y aún por las comunidades. En los hechos ha derivado en una enseñanza diferenciada para quienes están en condiciones de acceder a la universidad y quienes estarían condenados a actividades de servicio. Las escuelas del segundo tipo rebajan las exigencias de contenido (y hasta de asistencia a clases), porque tampoco tendrían las condiciones mínimas para otro tipo de logros. Es el sistema educativo funcionando ya no como formador sino como contención social. En esa enseñanza secundaria, a la larga, la enseñanza de la filosofía estaría condenada a desaparecer. De hecho las reformas implantadas en Latinoamérica en los 90, bajo el apadrinamiento de organismos financieros internacionales, han propuesto curriculas donde la enseñanza de la filosofía casi no aparece.

\section{¿Hay alternativa?}

La pregunta clave es hoy, ¿existe frente a la pedagogía light, neo-conservadora y estratificada otra alternativa que rejerarquice la educación en general y la enseñanza de la filosofía en particular? ¿Puede existir una tal posibilidad en medio de las condiciones descriptas?

Desafortunadamente, mi parecer es antes que nada: que no hay una respuesta segura para esta pregunta crucial. La educación en general y la enseñanza de la filosofía como "barómetro" privilegiado de ésta, son enormemente sensibles a múltiples factores sociales, económicos, políticos, culturales e ideológicos, cuyo desarrollo no puede ser previsto.

Sin embargo hay algunas cosas que se pueden decir, y algunos hechos que permitirían albergar ciertas esperanzas.

En primer lugar: es de fundamental importancia que tomemos conciencia de toda esta compleja situación y la repensemos constantemente en sus múltiples dimensiones y factores. Esto también es una tarea educativa y filosófica. 
En segundo lugar, aún en esta situación con rasgos tan adversos, pueden verse algunos signos que podrían dar lugar a un giro dialéctico hacia un reposicionamiento democrático-progresista de la educación en general y de la enseñanza de la filosofía en particular. ¿Cuáles serían esos signos alentadores?

En primer lugar el modelo unipolar y estratificante de globalización que imperó en los 80 y 90 parece estar llegando a su agotamiento. Diversos indicadores políticos, económicos y hasta geopolíticos que no vamos a analizar, parecen estarlo indicando.

En segundo lugar y muy vinculado con lo anterior, en América Latina parece estarse verificando una nueva alianza de grupos sociales a la interna de los países, donde los empresarios nacionales buscan un cierto desarrollo nacional que les permita aliviar la competencia de las trasnacionales y el ensanchamiento de los mercados nacionales. Eso requeriría hacer una apuesta fuerte y autónoma a la educación, como la que se produjo a comienzo de siglo $\mathrm{XX}$ durante el proceso de sustitución de importaciones, con la consiguiente revalorización social de la educación y de la figura docente.

En tal eventualidad la enseñanza de la filosofía tiene un papel importante a cumplir y los aspectos administrativos deberán volver a estar supeditados a los aspectos educativos. En el contexto de un tal proyecto nacional, también los medios masivos y la esfera política recobrarían un protagonismo y un papel de agentes culturales que han perdido.

En tercer lugar, el colapso provocado del welfare-state tiende a generar formas alternativas de solidaridad (cooperativas, autogestión, etc.) y proyectos colectivos, que requieren de un replanteamiento general de la producción, la economía y los vínculos humanos donde también la filosofía tiene un rol fundamental

En cualquiera de las dos alternativas anteriores - proyecto nacional o formas solidarias de emergencia - las adversas condiciones anímicas de: depresión, individualismo, nihilismo e inmediatismo, comienzan a revertirse y a posibilitar una actitud de cuestionamiento y argumentación profundizada del tipo que nuestra disciplina necesita y canaliza.

Es importante señalar que esto no es mera "imaginería". En mi tarea de supervisor docente por todo Montevideo ya he podido observar que en los institutos cercanos a los barrios cooperativos -que, además de construir cooperativamente la vivienda, propician bibliotecas, videotecas, ciclos culturales y cooperativas de consumo - se observa una mayor motivación, una mejor instrumentación y hasta una mayor creatividad.

En cuarto lugar, y aún cuando las previsiones anteriores - en las que no soy nada experto - estuvieran equivocadas, la dinámica económica y tecnológica general de la llamada sociedad posindustrial requiere una fuerte inversión en educación y en una educación que permita la adaptación a condiciones cambiantes. Eso sólo se puede lograr en el mediano plazo por la potenciación de las capacidades 
instrumentales y la cultura general, en cuyo caso la enseñanza de la filosofía deberá ser nuevamente rejerarquizada.

\section{¿Qué podemos hacer?}

Examinado, aunque sea someramente, el contexto socio-cultural que rodea la educación media en general y la enseñanza de la filosofía en particular, la siguiente y decisiva cuestión sería: ¿Qué podemos hacer -o que debemos proponer- como profesores secundaristas de filosofía para que este vital aprendizaje se torne viable y retome un nivel que nunca debió haber perdido?

Como mi tesis general es que la enseñanza de la filosofía es altamente sensible a las condiciones en que se desarrolla la educación media en general, debo distinguir aquí entre propuestas que hacen a la educación media como un todo y propuestas que hacen a la enseñanza filosófica en particular.

En relación a lo primero parece que deberíamos reivindicar:

$\left.1^{\circ}\right)$ Una educación media básica sólida, no deprivada de contenidos ni de exigencias lingüísticas y de estrategias básicas de razonamiento. Si esto no se diera -como algunos estudios están indicando que ocurrió a consecuencia de las reformas light de los 90'- los estudiantes accederían al segundo ciclo de enseñanza secundaria, donde aparecen los cursos de filosofía, sin los conocimientos ni las capacidades instrumentales indispensables para el trabajo en filosofía.

$2^{\circ}$ ) Dentro de las normas de la participación democrática, los profesores de filosofía, y en particular los de cierto nivel de actualización, deberían usar su capacitación para evaluar y potenciar grupalmente el valor cultural y educativo no sólo de las nuevas reformas educativas sino todos los espacios de los medios masivos que puedan hacer un aporte en ese sentido (radio, TV, periódicos, bibliotecas, discusiones públicas y debates parlamentarios, etc.). Ésto, además, crearía una serie de nuevos frentes ocupacionales para los egresados de estudios filosóficos, que también contribuiría a subir la percepción de la actividad.

Respecto a la enseñanza media de la filosofía en particular, y sin entrar en detalles técnico-didácticos que son conocidos, considero importante:

$\left.1^{\circ}\right)$ Nos sigue pareciendo pertinente nuestra propuesta de 1988 sobre lo que llamábamos "los grandes temas de nuestro tiempo".

Sin perjuicio de los temas y los momentos fundamentales de la tradición de la filosofía - sin la cual todo lo actual pierde su real dimensión histórica - creemos que buena parte de los cursos de filosofía deberían estar dedicados al análisis filosófico de los grandes temas de nuestro tiempo.

Entendemos como "grandes temas de nuestro tiempo" a los fenómenos teóricos o prácticos que están haciendo la historia de nuestra época. Los grandes fenómenos que están generando las condiciones del futuro de la humanidad y de nuestra 
América. Problemas del conocimiento o de definición axiológica que marcan a este tiempo y al porvenir. Problemas en fin que tienen que ver con el posible sentido de la vida de los jóvenes y adultos que en este tiempo vivimos y actuamos.

Pensamos, por ejemplo, en el tema de los cambios de costumbres y de valores, en el tema de la aceleración histórica, en el de la globalización, en el de la revolución científico-tecnológica, en los temas de los medios masivos, de la justicia en un mundo de crecientes desigualdades, en la epistemología de las ciencias humanas y la irrupción de nuevas corrientes y valores artísticos y en los temas de la violencia, la guerra y la paz.

Esto no implica diluir la especificidad del análisis filosófico, transformando nuestro papel en algo así como compensadores de cultura general, o de meros reiteradores de temas cubiertos por las ciencias naturales o sociales. Por el contrario, reivindica el papel universal del análisis y la reflexión filosófica; cualquiera de estos temas, sea cual sea su naturaleza y la disciplina específica que lo aborda científicamente, deja un margen para la reflexión filosófica, nunca agotado por la ciencia correspondiente. Pues, dicho en forma breve, el análisis que haremos desde la perspectiva, la especificidad y el nivel filosófico no se limitará al cómo y al por qué si no que buscará el sentido y la proyección de los hechos y los problemas, revisará los fundamentos epistemológicos de las elaboraciones teóricas o analizará críticamente el sentido de nuestras costumbres, concepciones y valores.

Así mismo, esta propuesta ha de poner especial cuidado en no desatender los grandes temas de la historia de la Filosofía, de la historia de la Ciencia o de la historia de la Cultura en general. Pues esta jerarquización de los temas de nuestro tiempo no debe conducir, de ningún modo, a una especie de "presentismo" que nos lleve a estar a la pesca de la última teoría o del último suceso; con lo cual, lejos de ubicarnos estaríamos perdiendo perspectiva y despreciando los legados históricos. Sería una posición realmente anticultural, desorientadora y superficial. Una buena formación histórica es imprescindible para ubicarse, para valorar y para comprender los fenómenos actuales.

$2^{\circ}$ ) Creemos conveniente dedicar al menos la mitad del último curso preuniversitario de Filosofía al análisis filosófico de temas vinculados a la disciplina universitaria que el estudiante se dispone a cursar. Así, por ejemplo, en los cursos pre-universitarios de Ingeniería se debería dedicar una unidad al análisis de los fundamentos de la Matemática o de la revolución paradigmática de la física relativista y/o cuántica, o la relación entre lógica y computación. En los cursos preuniversitarios de Medicina se podrían ver temas vinculados con la ética médica y la ingeniería genética. En los cursos de Derecho deberían incluirse unidades sobre la fundamentación filosófica de los sistemas jurídicos y del derecho internacional o los temas de la actual polémica sobre la justicia.

Esta idea tiene varios fundamentos. Señalemos uno solo: el estudiante debe sentir que la filosofía, además de cultura general y cierta apertura mental, le dio algunos fundamentos para pensar temas que lo han de acompañar durante toda su vida profesional y laboral. 
$\left.3^{\circ}\right)$ Parece conveniente proponer modalidades más constructivas y personales de evaluación que tiendan a sustituir el clásico examen total que puede ser resuelto por la mera apelación a la memoria. Concretamente, estamos pensando en la elaboración de pequeños ensayos a la manera anglosajona, donde el alumno deba enfrentar de modo personal pero documentado y argumentativo un problema concreto vinculado con los temas del programa que luego será corregido por otro docente o tribunal. Esta modalidad de evaluación tiende a asegurar un abordaje más problematizador y argumentativo de los temas y a requerir una apropiación más personal de los conocimientos y habilidades implicadas, a la vez que quiebra la oposición - de matriz autoritaria - entre alumno evaluado - docente evaluador para unirlos tras un esfuerzo común y gradual.

Como se ve, estos tres primeros criterios apuntan a conseguir una revalorización de la tarea filosófica por parte de los alumnos.

$\left.4^{\circ}\right)$ En cuarto lugar, y muy vinculado con las tres propuestas anteriores, deberíamos dar un lugar especial a tareas filosóficas de perfil interdisciplinario que rejerarquicen la asignatura en el contexto general del currículo, sobre todo a nivel pre-universitario.

Casi todos los temas pre-profesionales que antes mencionábamos se prestan a este tipo de abordaje. Se ha experimentado, por ejemplo, en trabajos interdisciplinarios con Historia sobre la metodología de las ciencias sociales,o, la incidencia de las condiciones e ideologías de los investigadores. Con disciplinas artísticas, se ha trabajado el tema de objetivismo o subjetivismo de la evaluación estética, etc. Con Derecho, se ha abordado el tema del derecho natural o del relativismo y el universalismo en la doctrina de los derechos humanos. E incluso, en temáticas de ciencias sociales sin contenidos explícitamente filosóficos, el docente de filosofía puede actuar como coordinador metodológico, supervisor del marco teórico y de la estructuración y coherencia general del trabajo. Para eso, él está especialmente capacitado y eso es absolutamente imprescindible.

Como se puede observar, si los tres primeros criterios se dirigen a conseguir una revalorización de los cursos de filosofía por parte de los alumnos, este cuarto criterio apunta además a rejerarquizar a la Filosofía en el contexto del currículo y en el seno mismo de la comunidad educativa. Es que: ninguna campaña "concientizadora" logrará convencer a la opinión pública ni a las autoridades de la importancia de la enseñanza media de la filosofía, si antes no se la hicimos sentir a los propios alumnos y a los propios colegas de otras asignaturas.

\section{Conclusión}

Digamos para concluir. si en la primera parte de esta exposición analizábamos una realidad de condiciones adversas, y en la segunda indicábamos la posibilidad de un giro dialéctico positivo, sobre el cual podemos tener alguna incidencia pero sólo parcial; en esta tercera parte hemos analizado aspectos que están a nuestro alcance y que pueden contribuir a un desenlace positivo de la tensión antes reseñada. 
Sin duda, lograr comprender y actuar sobre todos estos aspectos en el contexto de las complejas realidades de los sistemas educativos y de las realidades actuales no es una tarea nada fácil. Deberemos impostergablemente aprender a actuar en forma grupal y cooperativa. Quizá...: superar el clásico individualismo de los filósofos sea el mejor resultado que nos espere del otro lado de tan compleja situación. En este sentido, vuestros encuentros e intercambios regionales, nacionales e internacionales, son, no solo pertinentes sino rigurosamente imprescindibles.

\section{Referências bibliográficas}

CONNOR, Steven. Cultura postmoderna (1989). Trad. Esp. Madrid: Akal, 1996.

FINKIELKRAUT, Alain. La derrota del pensamiento. Barcelona: Anagrama, 1990.

GIROUX, Henry. Los profesores como intelectuales. Hacia una pedagogía crítica del aprendizaje (1988). Trad. Esp. Barcelona: Paidós, 1990.

JAMESON, Fredric. El postmodernismo o la lógica del capitalismo avanzado. Buenos Aires: Piadós, 1992.

NAVIA, Ricardo. La enseñanza media de la filosofía y los grandes temas de nuestro tiempo, Rev. Reflexào, Puccamp, n 43, Campinas, 1989, pp.26-34.

OBIOLS, Guillermo; Di Segni, S. Adolescencia, posmodernidad y escuela secundaria. Buenos Aires: Kapelusz, 1996.

P.I.S.A.-O.E.C.D. Programme for International Student Assessment. In: http://www.pisa.oecd.org/

SARLO, Beatriz. Instantáneas. Medios, ciudad y costumbres en el fin de siglo. Buenos Aires: Ariel, 1996.

(1) Professor de la Universidad de La República de Uruguay. Correo electrónico: naviamar@adinet.com.uy 